Témoigner Témoigner. Entre histoire et mémoire

Getuigen Revue pluridisciplinaire de la Fondation Auschwitz

$125 \mid 2017$

Histoire et mémoire de la persécution des

homosexuel-le's par les nazis

\title{
L'histoire des 3 Adolf : le chant du cygne du dessinateur de manga Osamu Tezuka
}

Message to Adolf: de zwanenzang van de Japanse mangatekenaar Osamu

Tezuka

\section{Brecht Capiau}

Traducteur : Emilie Syssau

\section{OpenEdition}

Journals

Édition électronique

URL : https://journals.openedition.org/temoigner/6267

DOI : 10.4000/temoigner.6267

ISSN : 2506-6390

Cet article est une traduction de :

Message to Adolf: de zwanenzang van de Japanse mangatekenaar Osamu Tezuka - URL : https:// journals.openedition.org/temoigner/6262 [nl]

Éditeur :

Éditions du Centre d'études et de documentation Mémoire d'Auschwitz, Éditions Kimé

Édition imprimée

Date de publication : 1 octobre 2017

Pagination : $32-35$

ISBN : 978-2-930953-01-4

ISSN : 2031-4183

Référence électronique

Brecht Capiau, "L'histoire des 3 Adolf : le chant du cygne du dessinateur de manga Osamu Tezuka », Témoigner. Entre histoire et mémoire [En ligne], 125 | 2017, mis en ligne le 24 décembre 2021, consulté le 04 février 2022. URL : http://journals.openedition.org/temoigner/6267 ; DOI : https://doi.org/ 10.4000/temoigner.6267 


\section{L'HISTOIRE DES 3 ADOLF : LE CHANT DU CYGNE DU DESSINATEUR DE MANGA OSAMU TEZUKA}

MANGA «A l'instar de la Neuvième Symphonie de Beethoven, L'histoire des 3 Adolf est l'œuvre d'un génie vieillissant dont la créativité parvenait encore à supplanter celle de ses contemporains. Personne hormis Tezuka ne pouvait mieux réunir ces nombreuses idées en gardant une cohérence ; personne ne pourrait esquisser autant de personnages et leur donner à chacun un but... II est difficile d'imaginer une autre œuvre de Tezuka aussi achevée que celle-ci. Note: A. » (Anime News Network)

e17 août 2010, l'hebdomadaire néerlandophone Knack dévoilait une première mondiale : le
journaliste Jean-Paul Mulders avait, après anajournaliste Jean-Paul Mulders avait, après ana«haplogroupe » du Führer et pouvait ainsi prouver qu étaient ses ancêtres. L'haplogroupe Elblb concorde de ( le deuxième haplogroupele plus fréquent chezles Juif (asheenazes. Voila quivenait raviver une vieillerumeur la usses l'autorisation d'analyse bout de tissu imprégné de sang chis bunker dinitler à Berlin. Depuis. trìs vrapenteste daire se sit heuté à un refus dest daire se soit heurte à un refus des inpos. croupe de poputation il à quetereupén nait précisément.

Ce n'est pas la première fois qu'historiens et journaliste prétendent qu'Hitler était juif En 1933 , lannée où Hitler a été Dily chirror a relaté l'histoire

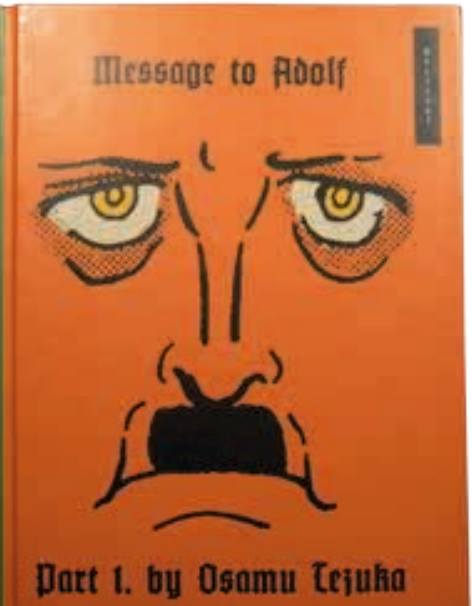

Datt 1. by Osamu Tejuka
L'HYPOTHĖSE DE FRANKENBERGER

L’histoire des 3 Adolfa étépubliée de 1983 à 1985 en livraisons hebdomadaires dans le magazine japonais Shasons hebdomadaires dans le magazine japonais Adolf $\mathrm{B}$. Adorf Hitler, le Funce dont lalliance et le lien spécia Kamil un Juif allo Adelf Kauf Juf allemand che landit a Kobe; et enfin Wolf Wous jous les suivons à travers les yeux de Sohei Toge, un

Août 1936, Berlin. Sohei Toge est envoyé à Berli afin d'y couvrir les Jeux olympiques d'été pour l'agence afin d'y couvrir les Jeux olympiques d'éte pour l'agence télép

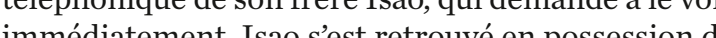
dow documents secrets qui, renduspubsics, pour raientprorendez-vous Sohe retrouve son fì tre trop tar

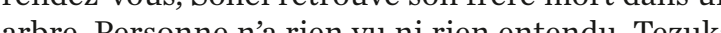
a indén pense Alf duitcheock Toutconé pense Alf nitch cock. Toutcon. dans La Mort aux trousses, Sohei est ici un Monsieur Tout-le-Mer ments se. Ondécourepres

Osamu Tezuka utilise l'hypothèse de Frankenberger pour point d'ancrage de son récit. Avec une subtilité ger pour point d'ancrage de son récit. Avec une subtilite médite, il fanchit la ligne mince entre fait et fiction pour ansi captiver le lecteur ennotionnellement et intellectuellement. Durant le process de Nuremberg ans Frank, dabord lavocat personnel d'Adolf Hitlan ilégitime de Maria An Schicklgruber et du fis de liégine de du sang juif coulait de-ci, Leopold Frankenberger du sang juif coulait don drans low plus tard démentiles déclarations Joachin Fest ont plust ler.

La recherche des documents, parmi lesquels le certificat de naissance d'Hitler est le fil conducteur récit de Tezuka. Le journaliste Sohei Toge finira par sacrifier presque tout ce qui lui est cher pour évite ue ces documents ne tombent aux mains des nazis. Le protagonist nous fat àcet égrd penser aulonceur d'alertecontemporain Edward Snowden. Fiction

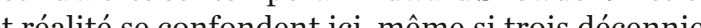

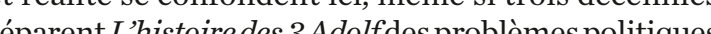
auxquels a été confronté Snowden.

KOBE, PORT D'ATTACHE SÛR POUR LES JUIFS

Adolf Kamil, le deuxième Adolf du récit, est un Jui Alemand qui estné et a grandià Kobe. Ses parents pos-

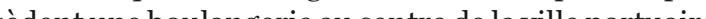
es Kamil minent une vie relativement confortable a Kobe, nêmequanda Seconde Guerremondialé́clate Ko 'A documents sur l'origne d'Hitler. C'est un des aspects les plus mar suats, moin

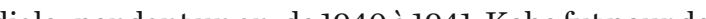
diale.pencint

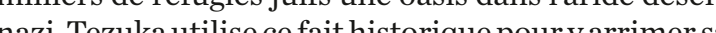

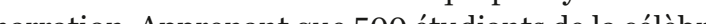
école to Apprenar que 500 étudiants de la célebre cutions, fui vers la Lituanie sous, à la suite de perséIsac Kamil, le pìre d'Adolf se rend aus sovietique, Isaac Kamil, le père dAdolf, se rend aussitot dans ce pays pour faire exacuer lè étudiants. Mais la Lituanie fort pour son altruisme.

CANDEUR ENFANTINE : ADOLF KAMIL CONTRE ADOLF KAUFMANN

Dans les années 1980, plusieurs pays commencent à adopter une attitude critique envers leur passé a adopter une attitude critique envers leur passe Requiem pour un massace (1985) offrè l'obsus un regard brut sur les atrocités nazies commises da le bloc de l'Est. Il était temps aussi pour le Japon de le bloc de lEst. Il était tenps aussi cour le Japon de sucioles sort à son passé guenier. Le Tombeau des toire d'un frèret un. Ce dessin animé raconte lhisculs au monde et doivent trouver leur chemin dan un pays détruit par un bombardement Ces deux films issus de deux cultures complètement différentes ont issus de deux cultures comṕétement diférentes ont en commun daborder les evénements depuis la perspective din entant, et de f'histoire des 3 Adolf de même 
- Tezuka estime que la solidarité et la compré hension entre des peuples différictions religienses différentes doivent être jeune ấ. Cela réduit les jeune âge. Cela reduit les tisques de discrimination et de racisme. Avecpour bel exemple lamitie entreAdolf Kamil et Adolf Kaufmann qui s’epanouit indépendan conviction politique. Il existe cen politique. férence évidonte entre les denx amis : Kamil, Juif alledeux anis : Kamil, Juif allejand, hequà il découve les japonaise oú il découvre les us et coutumes des Japonais, tandis qu'Adolf Kaufmann tis de lambassadeur allelégiés et pattie des priviprivée. Mais on lui cherche prive. Mais on lui chers' bientôt querelle. Kamil s'interpose alors et sinstaure en protecteur de Kaufnann entre les deux garçons nait ne amitie solide. Lorsque à son fils Adelf de fréquenà son fils Adolf de fréquenter Kamil parce qu'il es juif, lenfant ne comprend pas son père. Les terme bépacis conne « race depassentsonentendenent. Kamil est avant tout un bo qui il peut jorr. qui il peut jouer. Lamitie

'emporte sur la politique.

Aussi longtemps que Kaufmann vit à Kobe sous l'aile protectrice de sa mère, elle aussi hostilea a la politique

raciale du père, et quili peut profiter d'une jeunesse insouciante avec Kamil, il n'y a pas d'ombre au tableau.

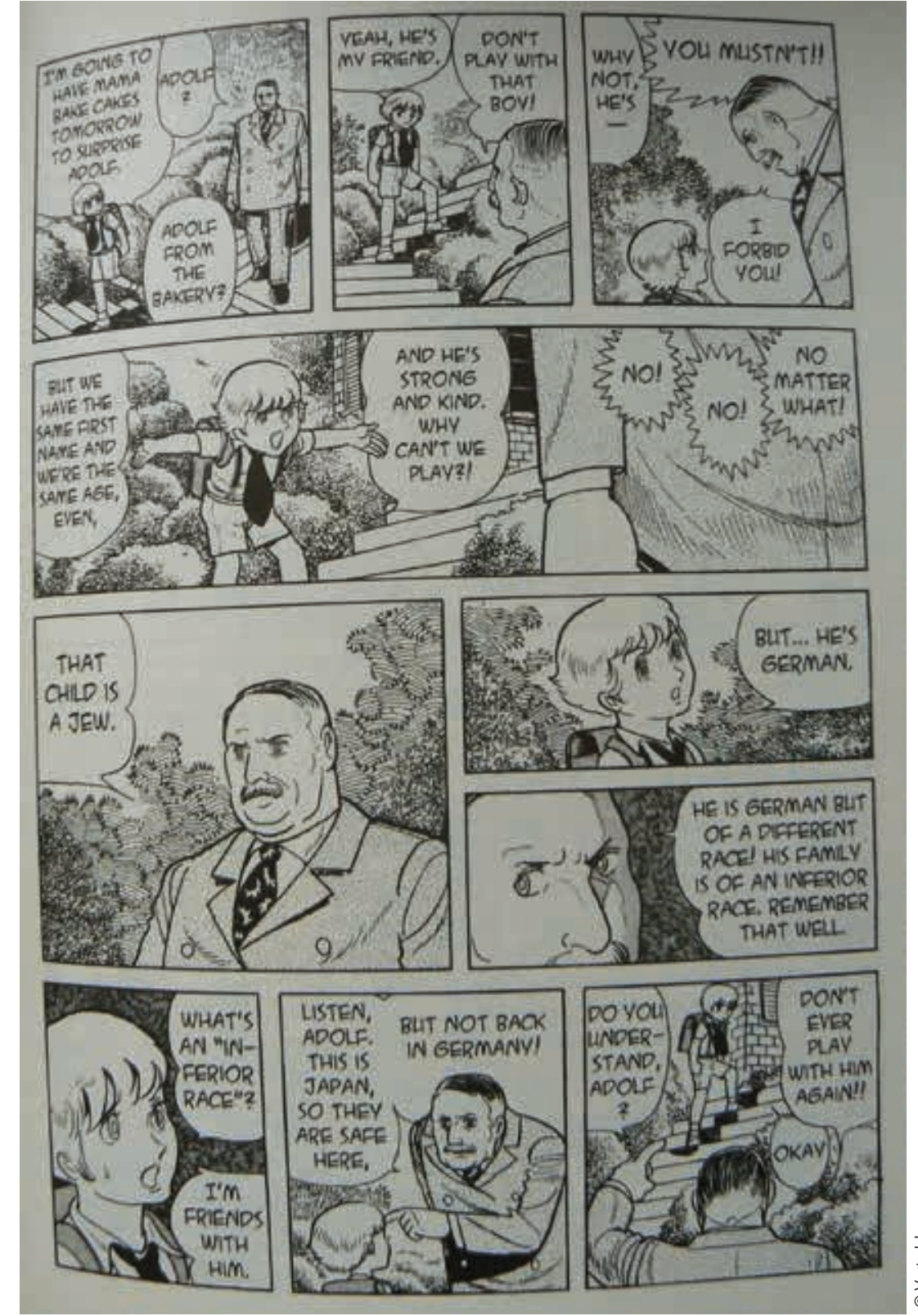

-Scène dans laquelle le père d’Adolf Kaufmann interdit
à son fils de continuer à fréquenter le Juif Adolf Kamil.

en Allemagne et être scolarisé dans une Adolf Hitler Schule, tout change. Adolf Kaufmann est endoctrin par l'idéologie nazie. Des années d'entraînement et

détude au sein des Hitlerjugend le rendent dur et font de lui un soldat idéal pour le Sicherheitsdienst. Kamil change aussi d'attitude à la suite du traitement brutal infligé à son peuple. Après la guerre, il se pose en champion du nouvel État d'Israël. Que sa création s’accompagne de victimes innocentes comme des femmes et des enfants est un prix qu'il est prêt à payer. Le lecteur continue à espérer que les choses vont s'arranger entre Kaufmann et Kamil, mais Osamu Tezuka montre qu'il n'y a pas de retour possible pour ces deux personnages, qui, chacun à sa manière, se radicalisent pendant les cinquante années couvertes par le manga.

\section{OSAMU TEZUKA ET SA POSTÉRITÉ}

Quand il a remporté le Inkpot Award lors du salon Comic-Con à San Diego en 2008, Tite Kubo, le concepComic-Con à San Diego en 2008, Tite Kubo, le conceptrès honoré, Osamu Tezuka ayant remportéce mêt prix hore, O Jap prix en 198. Mais me dans un public dexperts des rthy, 2009, p. 8)

Cette anecdote d'Helen McCarthy, autoritébritannique en matière de mangas et de dessins animés, en dit tions. d'ort après la Seconde Guere

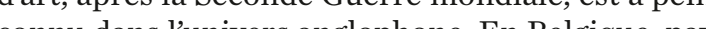

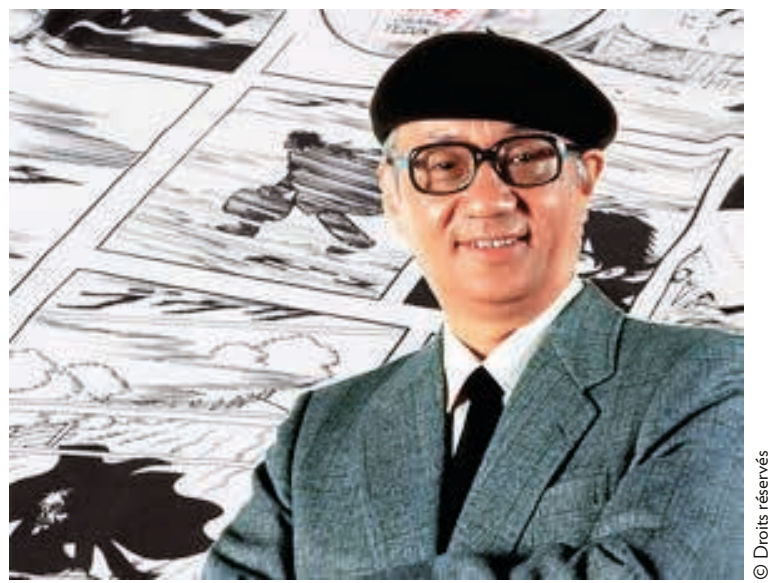

Osamu Tezuka

de la bande dessinée au même titre que la France et es États-Unis, ce n'est guère mieux. Avec L'histoire des 3 Adolf pourtant Osac mis. Á beaucoup ont échoué avant lui. Il réu rit l'Orient locident dans une bande dessiné hur lonient et loccident dans une bande dessiné híp chosich qu’un àlasoling Brecht Capiau $\rightarrow$ Bibliographie

$\diamond$ s.n., « Hitler was verwant met Somaliërs, Berbers en Joden » [Hitler était parent des Somaliens, Berbères et Juifs]. Knack, 17 août 2010 http://www.knack.be/nieuws/wetenschap/hitler-was-verwant-met-somaliers-berbers-en-joden/article-normal-10083.html, consultéle er mars 2017.

$\diamond$ McCarthy, Helen (2009): The Art of Osamu Tezuka, New York, Abrams ComicArts, 2009.

$\diamond$ Tezuka, Osamu: Lhistoire des 3 Adolf. Tonkam, « Tsuki Poche », 4 volumes parus de 1998 à 2001 